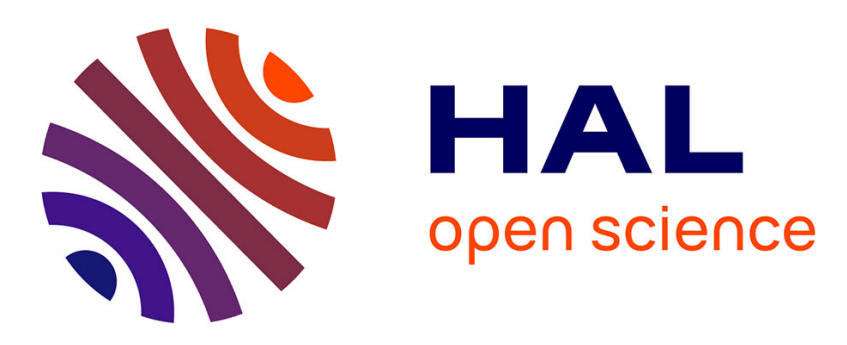

\title{
Vorticity and convective heat transfer downstream of a vortex generator
}

\author{
Thierry Lemenand, Charbel Habchi, Dominique Della Valle, Hassan \\ Peerhossaini
}

\section{- To cite this version:}

Thierry Lemenand, Charbel Habchi, Dominique Della Valle, Hassan Peerhossaini. Vorticity and convective heat transfer downstream of a vortex generator. International Journal of Thermal Sciences, 2018, 125, pp.342-349. 10.1016/j.ijthermalsci.2017.11.021 . hal-02527890

\section{HAL Id: hal-02527890 \\ https://univ-angers.hal.science/hal-02527890}

Submitted on 1 Apr 2020

HAL is a multi-disciplinary open access archive for the deposit and dissemination of scientific research documents, whether they are published or not. The documents may come from teaching and research institutions in France or abroad, or from public or private research centers.
L'archive ouverte pluridisciplinaire HAL, est destinée au dépôt et à la diffusion de documents scientifiques de niveau recherche, publiés ou non, émanant des établissements d'enseignement et de recherche français ou étrangers, des laboratoires publics ou privés. 


\title{
Vorticity and convective heat transfer downstream of a vortex generator
}

\author{
Thierry Lemenand $^{\mathrm{a}}$, Charbel Habchi ${ }^{\mathrm{b}}$, Dominique Della Valle ${ }^{\mathrm{c}}$, Hassan Peerhossaini ${ }^{\mathrm{d}, *}$ \\ ${ }^{a}$ LARIS EA 7315, Angers University - ISTIA, Angers, France \\ ${ }^{\mathrm{b}}$ Notre Dame University - Louaize, Mechanical Engineering Department, Zouk Mosbeh, Lebanon \\ c ONIRIS, Nantes, France \\ ${ }^{\mathrm{d}}$ Université Paris Diderot, Sorbonne Paris Cité, Energy Physics Group - AstroParticles and Cosmology Laboratory (CNRS UMR 7164), Paris, France
}

\begin{abstract}
A B S T R A C T
Vorticity generation has been identified, since the 80 's, as an efficient means for enhancing heat transfer; the mean radial velocity component due to the induced flow pattern contributes to the heat removal. In the present work, momentum and heat transfer are studied in a test section designed to mimic the industrial HEV (HighEfficiency Vorticity) mixer. It consists of a basic configuration with a unique vorticity generator inserted on the bottom wall of a heated straight channel. The aim of this work is to analyze to which extend the convective heat transfer is correlated to the vorticity, as it is presumed to cause the intensification. In this case, the driving vorticity is the streamwise vorticity flux $\Omega$, and the heat transfer is characterized by the Nusselt number $N u$, both quantities being spanwise averaged. The study is mainly numerical; we have used the previous PIV measurements and DNS data from the open literature to validate the numerical simulations. It is shown that there exists a strong correlation between the vorticity flux and Nusselt number close to the vortex generator. However, the axial variation diverges for these quantities when moving downstream. The Nusselt number presents a sharp peak over the VG and decreases nearly to its basic level just behind the VG, while the vortex persists far downstream from the tab and relaxes very slowly. Heat transfer intensification at the Nusselt peak is about $100 \%$, and reduces to about $6 \%$ downstream of the VG, the intensity of the vorticity momentum being decreased only to about $50 \%$ of its peak value at the test section outlet.
\end{abstract}

\section{Introduction}

Vorticity is an inherent feature of fluid flow and is often considered as an efficient mechanism in the heat and mass transfer phenomena. In many situations of practical interests vorticity is artificially generated to enhance heat and mass transfer [1-10], but also vorticity exists naturally in many types of fluid flows such as in the near-wall region of turbulent boundary layers $[11,12]$ or surfaces with curvature and/or rotation [13-15]. Physical understanding of the mechanism of heat transfer by vorticity is therefore crucial for active and passive control in numerous technological applications [16-20]. However, most of previous studies consider a global relationship between the velocity field and heat transfer coefficient, and little attention was paid to the role of the vorticity intensity.

Some studies investigated the analogy between the heat transfer and different flow parameters describing the strength of secondary flow such as the vortex circulation and vorticity flux. It was shown from experimental studies by McCroskey [21] that the theoretical predictions of the temperature-vorticity analogy agree well with the experimental results in laminar flows, but the theory failed in the transitional and turbulent flows. Song and Wang [22] defined a dimensionless secondary flow intensity $(\mathrm{Se})$ that is the ratio of inertial force to viscous force induced by the characteristic velocity of secondary flow to study the relation between vorticity and heat transfer in laminar flow. They obtained a fair correlation between the local and global dimensionless secondary flow intensity and the Nusselt number. Chang et al. [23] suggest the use of the span-averaged absolute streamwise vorticity flux to characterize the intensity of the secondary flow produced by vorticity generators. It was shown qualitatively that a similar behavior is observed between the longitudinal variation of the streamwise vorticity flux and the span-averaged Nusselt number downstream from the vortex generators. Actually, this spanwise-averaged absolute streamwise vorticity flux characterizes the convective heat transfer caused only by longitudinal vorticity, and does not account for the effects of transverse vorticity. Actually, it was shown $[2,24]$ that most of the heat transfer enhancement is caused essentially by the streamwise vortices, while the transverse stationary vortices, e.g. the wake recirculation, are globally "hot fluid traps", that poorly exchange.

\footnotetext{
* Corresponding author.

E-mail address: hassan.peerhossaini@univ-paris-diderot.fr (H. Peerhossaini).
} 


\author{
Nomenclature \\ $D_{h} \quad$ channel hydraulic diameter, $\mathrm{m}$ \\ $h \quad$ vorticity generator (tab) height, $\mathrm{m}$ \\ $J \quad$ streamwise vorticity flux, $\mathrm{s}^{-1}$ \\ $k$ turbulent kinetic energy (TKE), $\mathrm{m}^{2} / \mathrm{s}^{2}$ \\ $\mathrm{Nu} \quad$ Nusselt number \\ $\mathrm{Nu}_{0} \quad$ Nusselt number for straight channel \\ $Q_{w} \quad$ wall heat flux, W/m $\mathrm{m}^{2}$ \\ Re Reynolds number based on channel hydraulic diameter, \\ $\operatorname{Re}=W D_{h} / v$ \\ $\operatorname{Re}_{h} \quad$ Reynolds number based on the tab height, $\operatorname{Re}_{h}=W h / v$ \\ $S \quad$ cross section area, $\mathrm{m}^{2}$ \\ $T(x, y) \quad$ local temperature, $\mathrm{K}$ \\ $\bar{T}_{\text {inlet }} \quad$ averaged temperature at the inlet, $\mathrm{K}$
}

$\begin{array}{ll}\bar{T}_{\text {outlet }} & \begin{array}{l}\text { averaged temperature at the outlet, } \mathrm{K} \\ U\end{array} \\ W_{0} & \begin{array}{l}\text { convective heat transfer coefficient, } \mathrm{W} / \mathrm{m}^{2} \mathrm{~K} \\ \text { mean flow velocity, } \mathrm{m} / \mathrm{s}\end{array} \\ x, y, z & \begin{array}{l}\text { Cartesian coordinates, } \mathrm{m} \\ y^{+}, y^{*}\end{array} \\ \text { dimensionless wall distance }\end{array}$

$\lambda$

$v$

$\Theta$

$\omega_{z}$

$\Omega$

\author{
turbulent kinetic energy dissipation rate, $\mathrm{m}^{2} / \mathrm{s}^{3}$ \\ fluid thermal conductivity, $\mathrm{W} / \mathrm{mK}$ \\ kinematic viscosity, $\mathrm{m}^{2} / \mathrm{s}$ \\ dimensionless local temperature \\ streamwise vorticity, $\mathrm{s}^{-1}$ \\ dimensionless streamwise vorticity flux
}

In the present study, the spanwise-averaged absolute streamwise vorticity flux is computed only with the longitudinal vortex component, and is used for the discussion on the relationship between the heat transfer and the flow structure. The aim is to assess whether, in the VG case, this parameter is a relevant criterion for the heat transfer intensification.

The test section used in this study is designed based on the industrial HEV (High-Efficiency Vorticity) static mixer [25] that is a well-studied device and is used as multifunctional heat exchangers/reactors [26,27]. This apparatus is being increasingly incorporated in process industry for its mixing and heat transfer capabilities.

Static mixers are composed of a series of identical stationary inserts (called elements) fixed on the inner wall of pipes, channels, or ducts. The role of the elements is to redistribute the fluid flow in the directions transverse to the main flow, that is the radial and tangential directions. Static mixers divide and redistribute streamlines in a sequential fashion using only the pumping energy of the flowing fluid. The inserts can be tailored and optimized for particular applications and flow regimes. Commercial designs typically use standard values for the different parameters that provide high performance throughout the range of possible applications. In the particular case of the HEV static mixer, it is composed of a tube equipped with a series of four trapezoidal vorticity generators attached on the wall. The presence of inserts produces a complex vortex system in which concomitant phenomena simultaneously enhance mass and heat transfer. The study of such a flow is difficult because the longitudinal evolution of the streamlines is drastically modified by the presence of the vorticity generators. The complete geometry of this type of static mixer being complex to study, a simplified design where only one vorticity generator is included is proposed in this work. This flow type mimics the main feature, i.e. pressure driven longitudinal vorticity, of many heat transfer devices such as multifunctional heat exchangers/reactors [26,27]. Studying a geometry equipped with only one vorticity generator allows analyzing the longitudinal evolution of the flow characteristics without the perturbations brought about by other VGs downstream of the studied VG.

Numerical simulations are performed with the ANSYS Fluent CFD software to compute the convective heat transfer and the vorticity flux. The present flow arrangement is of particular interest since it allows studying this complex relationship in a vortical flow for which we can readily quantify the vorticity distribution.

Section 2 elaborates on the numerical procedure and experimental validation. In section 3 , results about the vortex strength and the temperature distribution are presented and the relationship between vorticity and heat transfer is discussed. The final section gives the concluding remarks.

\section{Numerical procedure}

\subsection{Physical domain}

The flow configuration studied here consists on a square duct flow of $7.62 \mathrm{~cm}$ each side, i.e. hydraulic diameter $D_{h}=7.62 \mathrm{~cm}$, and $33.15 \mathrm{~cm}$ long, in which a vorticity generator of trapezoidal shape is inserted on the bottom wall with an inclination angle of $24.5^{\circ}$ relative to the wall plane. The leading edge of the vorticity generator is located at $z=13.0 \mathrm{~cm}$. The dimensions of the vortex generator and the duct used here are adopted from Yang et al. [28] and Dong and Meng [29] for the sake of comparison and validation of the present numerical results with these previous studies. The dimensions of the physical domain and of the vorticity generator are schematically shown on Fig. 1 in the Cartesian frame of reference. In the following sections all spatial scales are scaled with the tab height $h=1.3 \mathrm{~cm}$. The vortex generator thickness is $0.5 \mathrm{~mm}$.

\subsection{Numerical method}

Numerical simulations in the present study are performed by the CFD code Fluent 6.3 [30]. The computational mesh is a cell-centered finite volume discretization. The conservation equations for mass, momentum, and energy are solved sequentially with double precision [31], segregated and second-order accuracy [32]. Pressure-velocity coupling is performed by finite volumes with the SIMPLE algorithm [33].

The choice of the RSM model - Reynolds stress model - is based on a previous study by Mohand Kaci et al. [34] who have tested different turbulence models to predict the flow dynamics in a HEV static mixer in the case of trapezoidal vortex generators. It was shown that the RSM model [35-37], associated with a two-layer model for the wall region computation, provides a satisfactory description of the flow pattern and turbulence statistics of the flow downstream multiple trapezoidal vortex generators. The Reynolds stress model requires a second order closure hypothesis as the Reynolds stresses are directly computed from the transport equations.

The flow in the near-wall region is computed by using a two-layer model. Following this model, in the viscous sub-layer, the one-equation model of Wolfstein [38] is used, in which only the turbulent kinetic energy transport equation is solved and the turbulent viscosity and energy dissipation rate are computed from empirical correlations based on length scales, given by Chen and Patel [39]. This two-layer model avoids the use of semi-empirical wall standard functions, which are not assessed for three-dimensional complex flows.

Similar numerical methods and turbulence model were used by several authors [40-44] to simulate the fluid flow and heat transfer in different geometries, including flow separation and shear flows which 


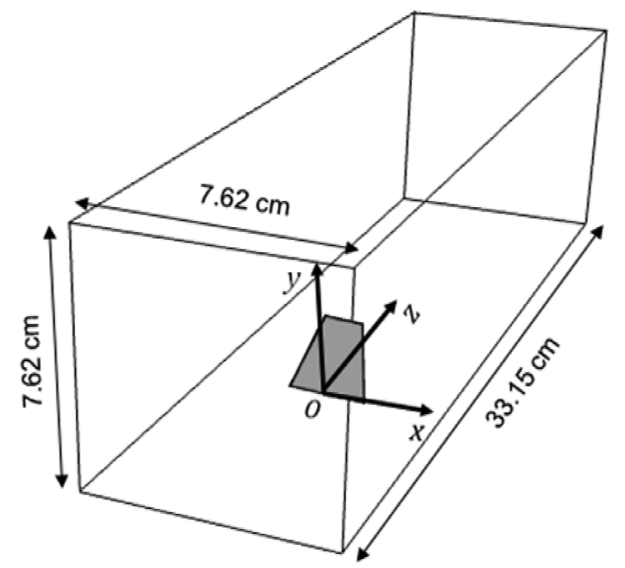

Fig. 1. Vorticity generator and duct dimensions represented on (a) global 3D view, (b) on the symmetry plane of the tab and (c) on a cross sectional plane.

(a)

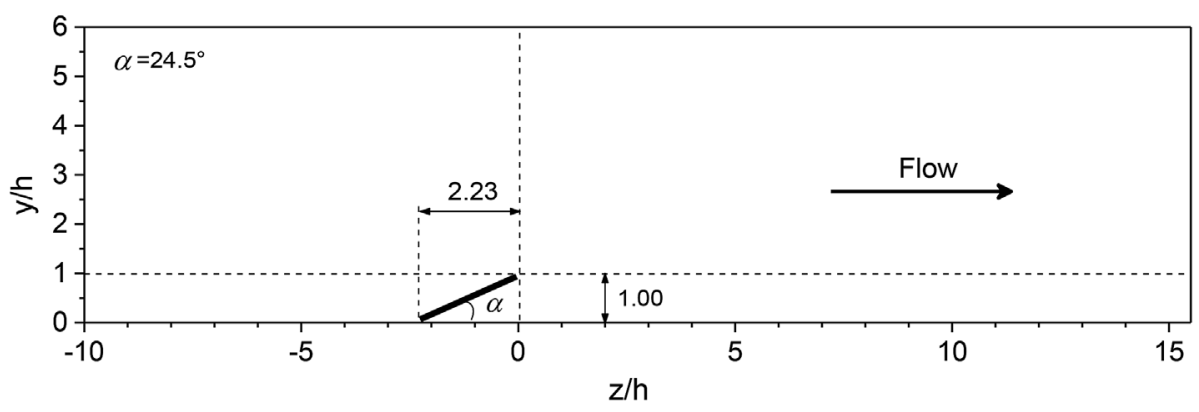

(b)

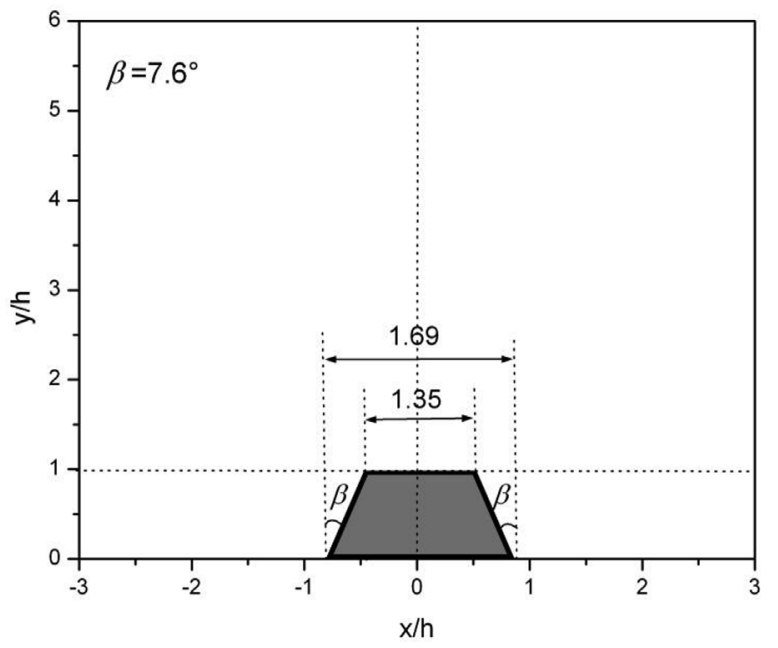

(c)

both are involved in the actual flow. These studies showed that the RSM model associated with a two layer model fairly predicts the experimental data.

\subsection{Boundary conditions}

The heat conduction inside the metallic tab is taken into account by using the coupled option of two-sided walls model with a small solid thickness. The thermal conductivity in the tab is taken constant and equal to $16.27 \mathrm{~W} / \mathrm{m}$.K corresponding to the conductivity of the steel.

The viscosity and the thermal conductivity, significantly change with the temperature. Therefore, these physical properties are assumed piecewise linear functions of temperature, as proposed by Rahmani et al. [41], and by using data from Lienhard [45]. Otherwise, the specific heat and density are assumed to be constant for the temperature range used here and their values are respectively set at $4182 \mathrm{~J} / \mathrm{kg} . \mathrm{K}$ and $998 \mathrm{~kg} / \mathrm{m}^{3}[41,45]$.

The dynamical boundary conditions are set by a no-slip condition at the walls, and a nil outlet pressure. A fully developed turbulent flow velocity profile at the inlet section imposes the flowrate. At this inlet, for numerical purposes, a given turbulent should also be specified, but these entrance conditions relax very quickly near the inlet and do not affect the computed turbulent field in the whole domain. In this instance, the turbulent kinematic energy (TKE) $k$ (eq. (1)) and its dissipation rate $\varepsilon$ (eq. (2)) are arbitrarily given by the turbulence intensity $I$ derived from the equilibrium turbulent flow [46]: 
$k=\frac{3}{2}\left(W_{0} I\right)^{2}$

$\varepsilon=\frac{C_{\mu}^{3 / 4} k^{3 / 2}}{\ell}$

with

$I=0.16 \mathrm{Re}^{-1 / 8}$

and $\ell$ is a characteristic length given by:

$\ell=0.07 D_{h}$

The fluid temperature at the inlet is set constant at $298 \mathrm{~K}$. Flow and heat-transfer simulations are carried out in a steady turbulent flow with a free stream flow velocity $W_{O}=0.16 \mathrm{~m} / \mathrm{s}$, which corresponds to a Reynolds number $R e=12100$, based on the channel height, and a Reynolds number based on the tab height $h$ of $R e=2080$, and for a constant wall temperature of $T_{w}=370 \mathrm{~K}$ applied to all the duct walls.

\subsection{Meshing and numerical solution accuracy}

A non-uniform unstructured three-dimensional mesh with hexahedral volumes is built and refined at all solid boundaries (using the software Gambit ${ }^{\circ}$ ). Mesh size is controlled by adjusting the number of the nodes in the $(x, y)$ directions, on the duct periphery, and on the axial coordinate $z$.

To determine the appropriate mesh density, the flow simulations are run with increasing mesh densities until no significant effect on the results is detected. The mesh validity verification is performed by using the method proposed by Roache [47] and Celik et al. [48], where the grid convergence index (GCI) and the apparent order of convergence (p) are obtained. The mesh validity verification is applied to the convective heat transfer coefficient $h$ and the streamwise helicity. It is found that the GCI did not exceed $2.3 \%$ while the order of convergence for the refined mesh case reaches about 3.2. The final mesh size after refinement is $1,800,566$. The maximum value of the wall dimensionless distance $y^{+}=u_{*} y / v$ (with $u_{*}$ the friction velocity at the wall) in the first grid point after refinement does not exceed 4. Since $y^{+}<5$, it is guaranteed that the viscous sub-layer is properly modeled.

Series of flow simulations are carried out for testing several stopcriteria values ranging from $10^{-3}$ to $10^{-9}$. It is found that beyond the value $10^{-6}$, no significant changes are observed in the temperature field and the turbulence kinetic energy, thus the value $10^{-6}$ is retained as the convergence criterion for the simulations.

\subsection{Experimental validation}

The simulations are performed in the same hydrodynamic conditions as previous PIV [28] and DNS [29] studies, so that the results of these studies can be used here to validate the present numerical results.

From Fig. 2 (a) the $y$ component, normal to the wall component, of the mean velocity $V / W_{O}$ at $y / h=0.5$ and $z / h=4$ is presented, showing a fair agreement between the experimental PIV measurements and DNS data. It is observed that the maximum normal velocity occurs at the tab symmetry plane due to the presence of the common up-flow induced by two neighbor counter-rotating vortices as will be detailed in the next section. The RSM simulations produce a zone of positive mean vertical velocity (upward flow) in the middle, and zones of negative values (downward flow) on both sides: these motions are induced by the streamwise rotation of the counter-rotating vortex pair and hairpin legs, discussed in section 3.1. The RSM simulations have captured this feature as well as the DNS simulations and the PIV measurements.

In Fig. 2 (b) the $(x, y)$ Reynolds stress component at $z / h=4.5$ is presented and compared with PIV measurements and DNS data. The main difference occurs in the shear region at $1.5<y / h<2$ where the Reynolds shear stresses are maximum. However, in the wake region $0<y / h<1.5$, the DNS results are far from PIV measurements and the present RSM simulations are closer to the experimental results, and in the region $y / h>2$ the three results are close from each other. The three methods captured the three distinct layers of Reynolds stress with alternating signs along the wall-normal direction.

Finally, it can be concluded that the present numerical simulations are in fair agreement with the flow dynamics in the studied geometry. The discrepancy observed between the three methods, PIV, DNS and RSM can be attributed to the lack of information in the numerical models and/or to experimental errors in the measurements inherent to PIV in low velocity zones. Moreover, in the DNS simulations the tab thickness is assumed negligible, which is not the case in the RSM simulation. The tab thickness may have a notable influence on the statistics by inducing additional instabilities and fluctuations near the tab edges and propagating downstream along the boundary of the wake.

\section{Results and discussion}

\subsection{Secondary flow pattern}

The flow streamlines on different cross sections downstream from the tab are represented in Fig. 3. For $z / h=2$ in Fig. 3 (a), a complex flow is observed consisting in two types of longitudinal vortices: primary counter-rotating vortex pair (PCVP) in the wake of the tab, and secondary corner vortices (SCV) in the corners of the channel.

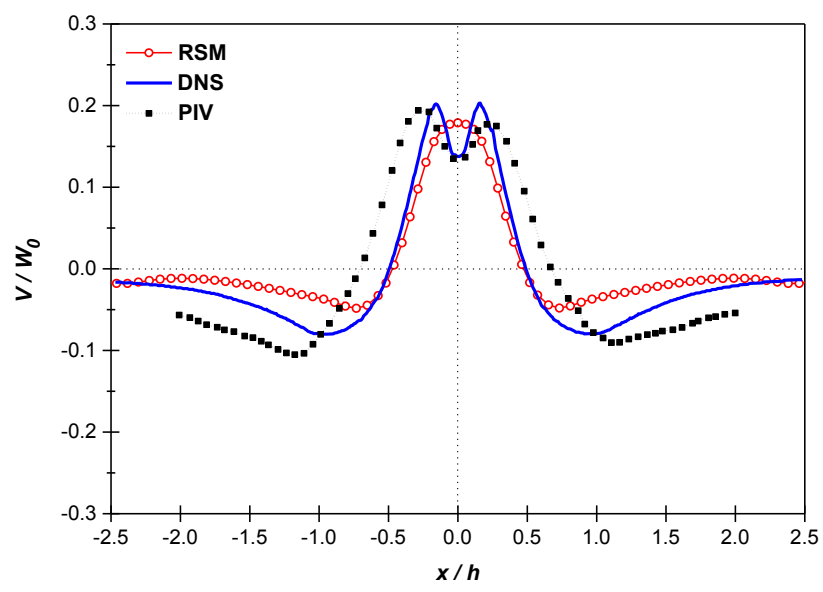

(a)

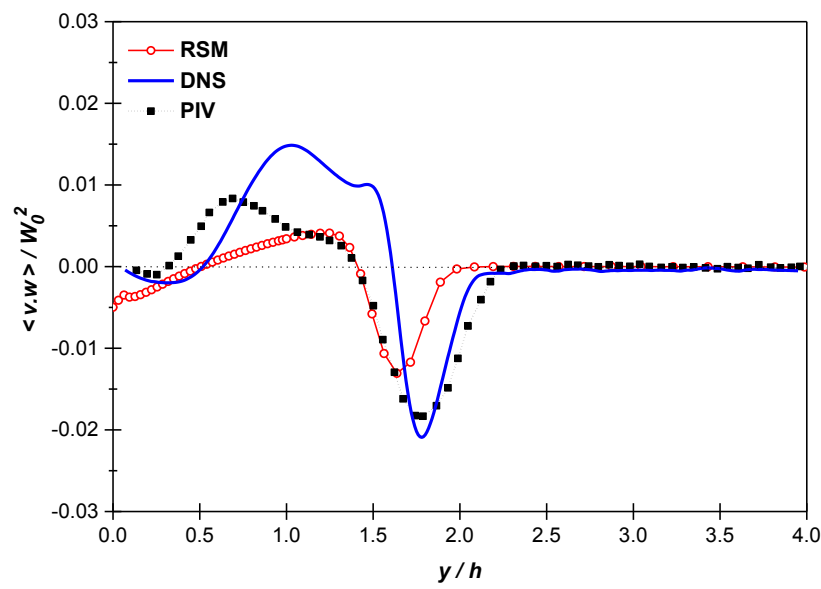

(b)

Fig. 2. Validation of the numerical results: (a) $y$ component of the mean velocity at $y / h=0.5$ and $z / h=4$, (b) the $(x, y)$ Reynolds stress component at $z / h=4.5$. PIV results are adapted from Yang et al. [28] and DNS results from Dong and Meng [29]. 

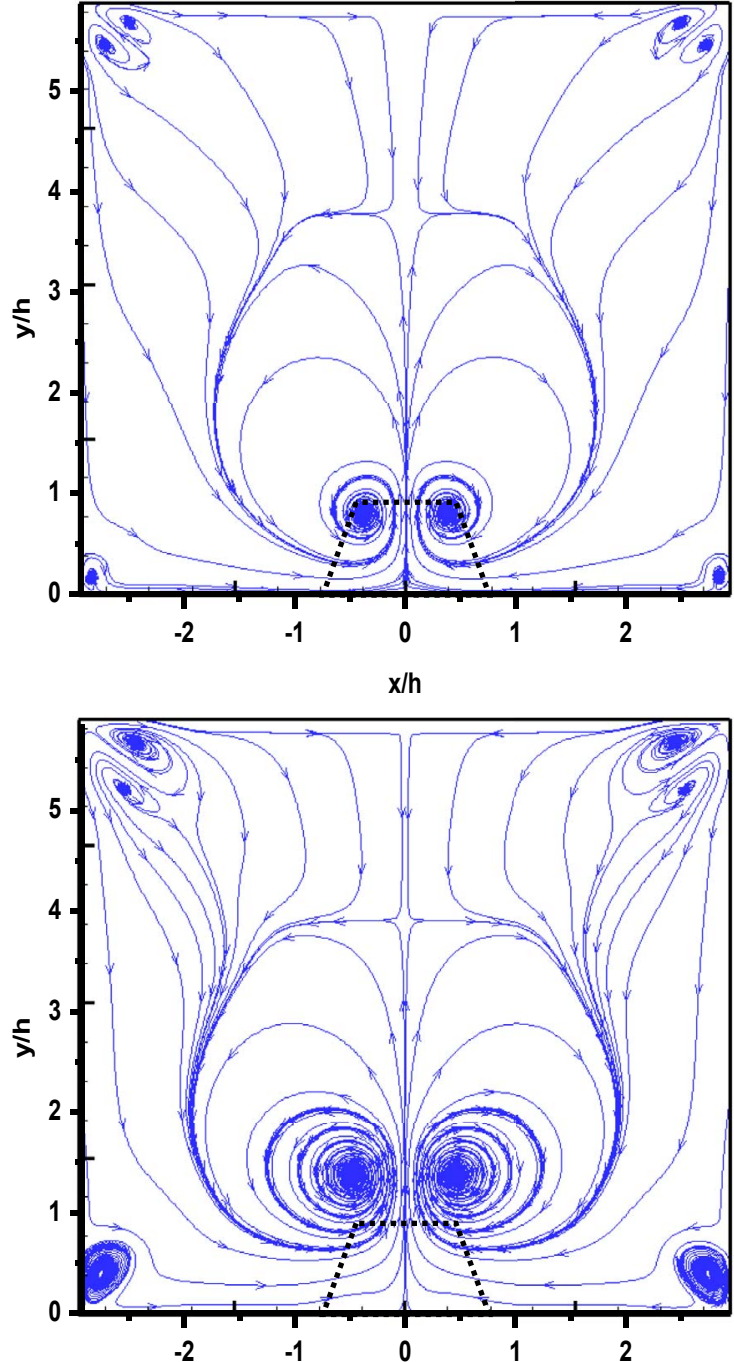

(b)

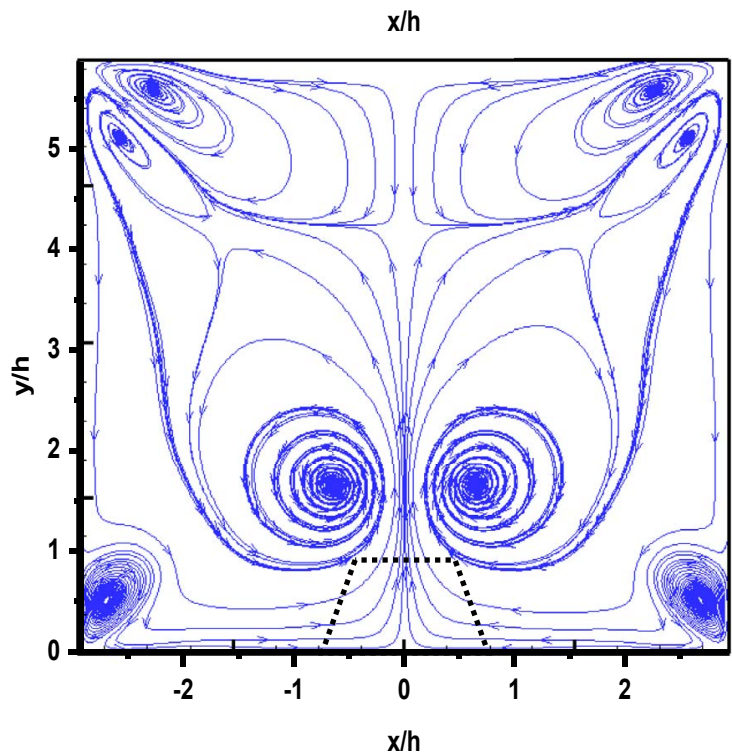

(c)

Fig. 3. Streamlines in the duct cross sections at (a) $z / h=2$, (b) $z / h=8$, (c) $z / h=15$ downstream from the vortex generator.

The PCVP are caused by the pressure difference between the high momentum fluid in the flow core, and the low momentum fluid in the tab wake. The common-up flow in the tab symmetry plane induced by

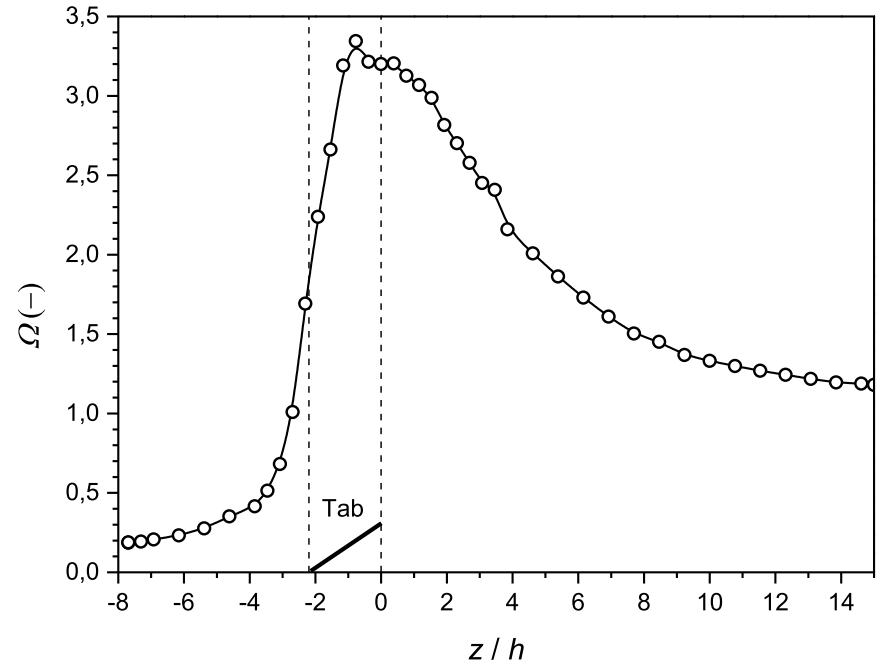

Fig. 4. Longitudinal evolution of the spanwise-averaged vorticity flux $\Omega$.

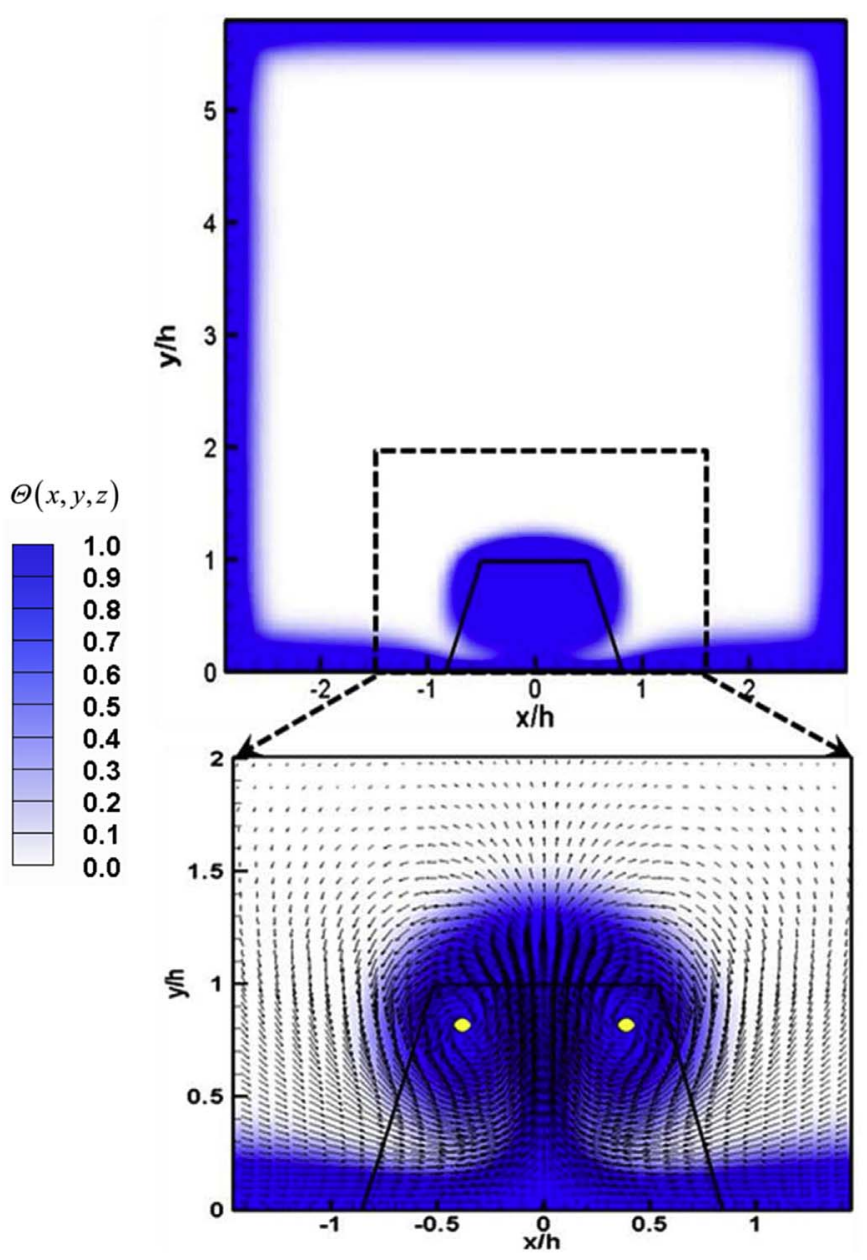

Fig. 5. Dimensionless temperature $\Theta(x, y, z)$ distribution in a duct cross section at $z / h=2$ downstream from the tab. The secondary velocity vector field is represented on the enlarging centered on the vortex generator. The yellow marked circles correspond to the vortex centers. (For interpretation of the references to colour in this figure legend, the reader is referred to the Web version of this article.)

the PCVP ejects the fluid particles from the wall region towards the flow core.

The SCVs are caused by the transverse gradients of the Reynolds shear stress components that influence the longitudinal shear stress of 


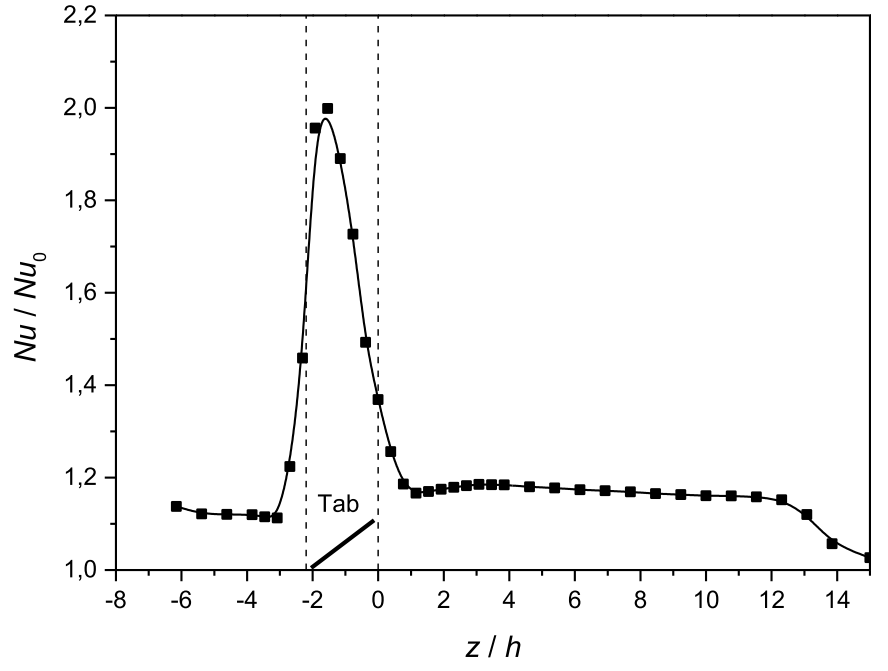

Fig. 6. Longitudinal evolution of the normalized Nusselt number $\mathrm{Nu} / \mathrm{Nu}$.

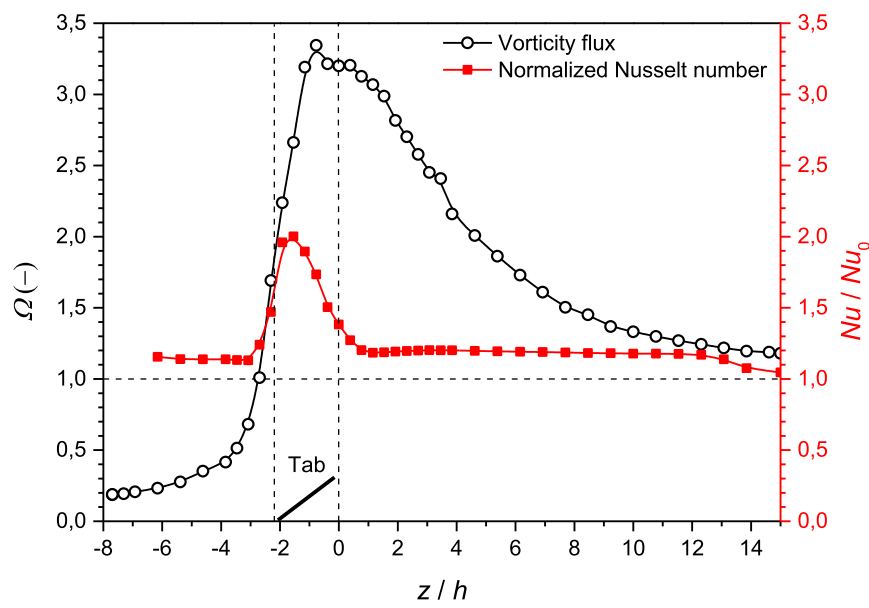

Fig. 7. Longitudinal evolution of the cross section-averaged vorticity flux $\Omega$ and normalized Nusselt number $\mathrm{Nu} / \mathrm{Nu}_{0}$.

the primary flow. This was shown experimentally by Gessner [49] and studied numerically by Husert and Biringen [50]. It has been observed that these vortices engulf high-momentum fluids in the flow core and transport them into near wall region, enhancing the heat and mass transfer phenomena [51]. The difference between the topology of the upper and lower SCV is caused by the presence of the PCVP near the bottom wall.

The streamwise evolution of these two types of longitudinal vortices can be observed by following Fig. 3 (a) to (c). For $z / h=8$ represented in Fig. 3 (b), and comparatively to $z / h=2$ represented in Fig. 3 (a), the upper and lower SCVs are getting larger when they move downstream from the tab. This phenomenon is amplified far downstream, as shown in Fig. 3 (c) for $z / h=15$. Another fact is that the centers of the PCVP migrate towards the flow core as one moves away from the tab. The low-pressure zone in the flow core that sucks up the PCVP causes this mechanism.

\subsection{Vortex strength}

In order to quantify the intensity of the secondary flow, we refer to the absolute spanwise-averaged streamwise vorticity flux $J$ defined by Chang et al. [23] and given in the following expression:

$J=\frac{\iint_{S}\left|\omega_{z}\right| d x d y}{\iint_{S} d x d y}$

where $\omega_{z}$ is the streamwise vorticity and $S$ the surface of the duct cross section. Here, a dimensionless value of the absolute cross-averaged streamwise vorticity flux, called hereafter vortices density, can be obtained from the following scaling:

$\Omega=\frac{J S}{W h}$

where $W$ is the mean flow velocity.

Fig. 4 represents the longitudinal evolution of the dimensionless vorticity flux. It appears that the peak value of $\Omega$, about 3.3, occurs at the location $z / h \approx-1.0$ corresponding to the abscissa of the middle of the tab. It can also be observed on Fig. 4 that the vorticity density upstream begins around 1.0 much before the tab, due to the presence of necklace vortices upstream the vorticity generator, as described by Habchi et al. [52]. Downstream, the vorticity density is decreasing rather slowly than the increase upstream.

\subsection{Temperature distribution}

With $T(x, y, z)$ the local temperature, $\bar{T}_{\text {inlet }}$ and $\bar{T}_{\text {outlet }}$ being the bulk temperatures on the inlet and outlet of the channel respectively, a dimensionless temperature $\Theta(x, y, z)$ is defined in Eq. (7), varying between 0 and 1 :

$\Theta(x, y, z)=\frac{T(x, y, z)-\bar{T}_{\text {inlet }}}{\bar{T}_{\text {outlet }}-\bar{T}_{\text {inlet }}}$

In Fig. 5, the temperature contour is plotted in the duct cross section

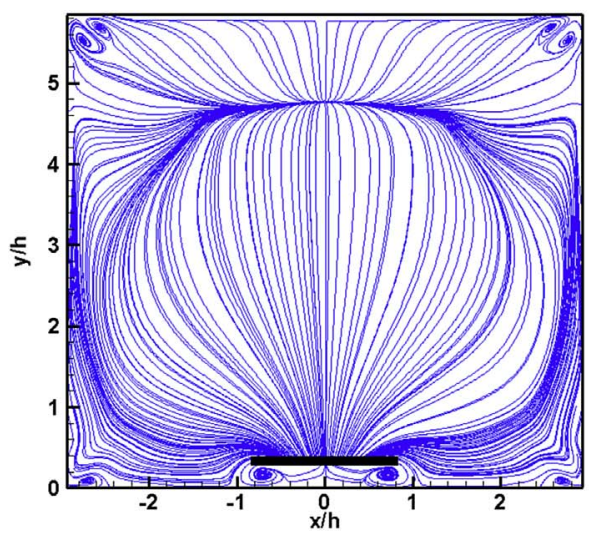

(a)

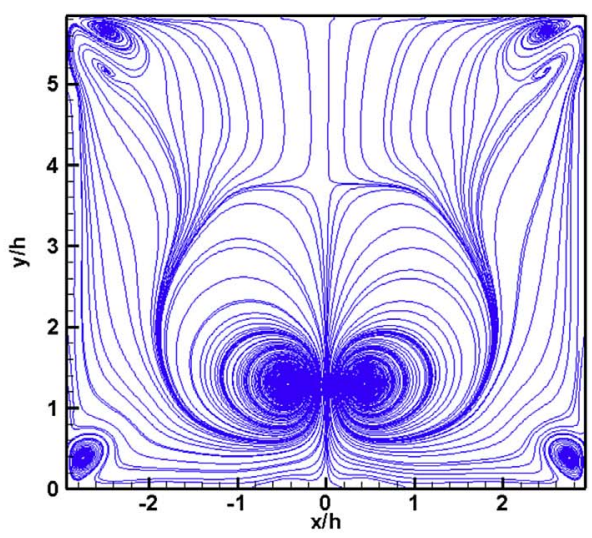

Fig. 8. Streamlines in the duct cross sections at (a) $z / h=-1.9$, and $(\mathrm{b}) z / h=6.9$ 
at $z / h=2$ on the same graph as the secondary velocity vector field. It can be observed that the heat is essentially transported by the commonup flow, in the tab plane of symmetry, towards the flow core. The hot fluid is homogenized into the PCVP, what it clearly shown by the mushroom shape of the contour. The SCV do appear to play a significant part in the heat transfer.

\subsection{Heat transfer}

The spanwise-averaged Nusselt number calculated by the expression below can characterize heat transfer:

$N u=\frac{U D_{h}}{\lambda}=\frac{\phi_{w}}{\left(T_{w}-T_{b}\right)} \frac{D_{h}}{\lambda}$

where $U$ is the spanwise-averaged convective heat transfer coefficient, $\lambda$ the thermal conductivity of the working fluid, $D_{h}$ the hydraulic diameter of the square duct, $T_{w}$ the wall temperature and $T_{b}$ the flow bulk temperature.

The Nusselt number is scaled by the Nusselt number for a straight channel without vorticity generator, $\mathrm{Nu}_{0}$ obtained from numerical simulations with identical conditions carried out in the same geometry of empty square duct flow.

Fig. 6 represents the longitudinal evolution of the normalized Nusselt number $\mathrm{Nu} / \mathrm{Nu}_{0}$. The maximum value for this ratio is about two and occurs at about the location $z / h \approx-1.0$ corresponding to the middle of the tab. One can observe on this profile that the heat transfer enhancement is mostly localized on the tab and sharply relaxes downstream, although the vortices are in their maximum strength. Usual consensus is that high heat transfer performance with low drag penalty occurs in the long distance downstream of VG since PCVP survives further downstream without attenuation. Actually the numerical results in Fig. 6 show that the ratio $\mathrm{Nu} / \mathrm{Nu} u_{0}$ increases from about 1.13 just upstream of the VG and remains around 1.20 in average in the range $1<z / h<12$ beyond which the values drop to unity: there is about $6 \%$ gain in heat transfer performance in the range $1<z / h<12$.

\subsection{Vortex strength and heat transfer}

Both axial profiles of the vortex strength and the heat transfer, $\Omega$ and $\mathrm{Nu} / \mathrm{Nu} u_{0}$ are plotted on Fig. 7. Both present a sharp increase just upstream the vortex generator, where the boundary layer is removed by the vortices and the local velocity takes a transverse component, as described by Habchi et al. [52]. The maxima of both profiles takes place at the tab location, the peak of vorticity being slightly shifted downstream compared with the peak of Nusselt number.

However, the behavior of $\Omega$ and $N u / N u_{0}$ downstream is rather different: the Nusselt number decreases immediately after the VG but $6 \%$ higher than upstream (till $z / h=12$ ), while the vortex persist far downstream from the tab (till $z / h=12$ ) and relaxes very slowly. This shows that the momentum transfer has higher Lagrangian persistence than the heat transfer. The complex structure of the vorticity hence presents a weak but interesting global effect on the heat transfer just after the VG. In fact, in the range $1<z / h<12$ (downstream of the tab) where the vorticity flux is intensified and is higher than its value upstream of the tab, one can observe about $6 \%$ increase on the Nusselt number compared to its values upstream of the VG. This interesting intensification is due to the high value of the vorticity flux.

It can be argued that the main effect is due to the acceleration of the fluid to the center when the flow meets the tab, combined with a very local contribution of the PCVP after it. This feature can lead to reconsidering the design of such heat exchangers in industrial devices, by choosing the tabs spacing based on the vorticity relaxation. Probably, a thermal optimization study based on a higher intensification on heat transfer could recommend shorter distance between the tabs, in spite of higher pressure drops, this distance could be chosen around the value $z$ / $h=12$.

To stress the highlighted behavior difference between the heat transfer and the momentum transfer, we have plotted on Fig. 8 the streamlines for two locations at $z / h=-1.9$ and $z / h=6.9$, which are measured at the same vorticity flux $\Omega=1.70$. Even though the vorticity flux has the same value, it clearly appears that the streamlines are totally different and can give an explanation of the behavior difference. Downstream from the tab (Fig. 8 (b)) the vorticity center is further from the channel wall than upstream of the tab (Fig. 8 (a)) and therefore, the Nusselt number is smaller in this case. It seems then quite evident that the rough comparison developed here does not take into account these topological characteristics of the secondary flow which have an essential importance on the convective heat transfer, thus the discrepancy in the values of the Nusselt number for a given vorticity flux.

\section{Conclusions}

A numerical study is performed in this work to investigate the momentum and heat transfers, by the vorticity strength $\Omega$ and the Nusselt number $\mathrm{Nu}$, downstream a single vorticity generator. Streamwise vorticity is generated in a turbulent straight channel flow by using a trapezoidal vorticity generator. It is observed that the profiles of $\Omega$ and $N u$ in the axial direction present peak in the tab section, but the spatial relaxation of the momentum is much slower than the heat transfer. Nusselt number decreases downstream of the VG, while the vortex persists far downstream from the tab and relaxes very slowly. Heat transfer intensification downstream of the vortex generator is about $6 \%$ over the high vorticity flux region.

This feature clearly shows that the intensification by the vortex generators technology requires a tailored design depending on the application, as the best spacing for mixing appears to be not same than for heat removal applications. For multifunctional heat-exchanger-reactors, an optimization procedure would be necessary to fulfill a high level of concomitant heat and mass transfers.

\section{Appendix A. Supplementary data}

Supplementary data related to this article can be found at http://dx. doi.org/10.1016/j.ijthermalsci.2017.11.021.

\section{References}

[1] S. Ferrouillat, P. Tochon, C. Garnier, H. Peerhossaini, Intensification of heat transfer and mixing in multifunctional heat exchangers by artificially generated stream-wise vorticity, Appl Therm Eng 26 (2006) 1820-1829.

[2] M. Fiebig, Vortices, generators and heat transfer, Chem Eng Res Des 76 (1998) 108-123.

[3] A. Ghanem, C. Habchi, T. Lemenand, D. Della Valle, H. Peerhossaini, Energy efficiency in process industry-high-efficiency vortex (HEV) multifunctional heat exchanger, Renew Energy 56 (2013) 96-104.

[4] C. Habchi, T. Lemenand, D. Della Valle, L. Pacheco, O. Le Corre, H. Peerhossaini, Entropy production and field synergy principle in turbulent vortical flows, Int J Therm Sci 50 (2011) 2365-2376.

[5] G. Zhou, F. Zhizheng, Experimental investigations of heat transfer enhancement by plane and curved winglet type vortex generators with punched holes, Int J Therm Sci 78 (2014) 26-35.

[6] N. Zheng, P. Liu, F. Shan, J. Liu, Z. Liu, W. Liu, Numerical studies on thermohydraulic characteristics of laminar flow in a heat exchanger tube fitted with vortex rods, Int J Therm Sci 100 (2016) 448-456.

[7] C. Habchi, T. Lemenand, D. Della Valle, H. Peerhossaini, Alternating mixing tabs in multifunctional heat exchanger-reactor, Chem Eng Process 49 (2010) 653-661.

[8] M. Oneissi, C. Habchi, S. Russeil, D. Bougeard, T. Lemenand, Novel design of delta winglet pair vortex generator for heat transfer enhancement, Int J Therm Sci 109 (2016) 1-9.

[9] T. Lemenand, P. Dupont, D. Della Valle, H. Peerhossaini, Comparative efficiency of shear, elongation and turbulent droplet breakup mechanisms: review and application, Chem Eng Res Des 91 (2013) 2587-2600.

[10] A. Khanjian, C. Habchi, S. Russeil, D. Bougeard, T. Lemenand, Effect of rectangular winglet pair roll angle on the heat transfer enhancement in laminar channel flow, Int J Therm Sci 114 (2017) 1-14.

[11] P.M. Le, D.V. Papavassiliou, A physical picture of the mechanism of turbulent heat 
transfer from the wall, Int J Heat Mass Transf 52 (2009) 4873-4882.

[12] J. Zhou, R.J. Adrian, S. Balachandar, Autogeneration of near-wall vortical structures in channel flow, Phys Fluids 8 (1996) 288-290.

[13] C. Habchi, T. Lemenand, D. Della Valle, H. Peerhossaini, Liquid-Liquid dispersion in a chaotic advection flow, Int J Multiph Flow 35 (2009) 485-497.

[14] T. Lemenand, C. Habchi, D. Della Valle, J. Bellettre, H. Peerhossaini, Mass transfer and emulsification by chaotic advection, Int J Heat Mass Transf 71 (2014) 228-235.

[15] R. Toe, A. Ajakh, H. Peerhossaini, Heat transfer enhancement by Görtler instability, Int J Heat Fluid Flow 23 (2002) 194-204.

[16] J.L. Aider, J.F. Beaudoin, J.E. Wesfreid, Drag reduction of a 3D bluff body using active vortex generators, Exp Fluids 48 (2010) 771-789.

[17] A. Ghanem, T. Lemenand, D. Della Valle, H. Peerhossaini, Static mixers: mechanisms, applications, and characterization methods-a review, Chem Eng Res Des 92 (2) (February 2014) 205-228.

[18] M. Hatami, D.D. Ganji, M. Gorji-Bandpy, Experimental investigations of diesel exhaust exergy recovery using delta winglet vortex generator heat exchanger, Int J Therm Sci 93 (2015) 52-63.

[19] C. Habchi, S. Russeil, D. Bougeard, J.-L. Harion, T. Lemenand, D. Della Valle, et al., Enhancing heat transfer in vortex generator-type multifunctional heat exchangers, Appl Therm Eng 38 (2012) 14-25.

[20] O. Lögdberg, K. Angele, P.H. Alfredsson, On the robustness of separation control by streamwise vortices, Eur J Mech B/Fluids 29 (1) (2010) 9-17.

[21] W.J. McCroskey, S.H. Lam, The temperature-vorticity analogy in boundary layers, Int J Heat Mass Transf 9 (1966) 1205-1214.

[22] K.-W. Song, L.-B. Wang, The effectiveness of secondary flow produced by vortex generators mounted on both surfaces of the fin to enhance heat transfer in a flat tube bank fin heat exchanger, J Heat Transf 135 (2013) 041902-041902-11.

[23] L.M. Chang, L.B. Wang, K.W. Song, D.L. Sun, J.F. Fan, Numerical study of the relationship between heat transfer enhancement and absolute vorticity flux along main flow direction in a channel formed by a flat tube bank fin with vortex generators, Int J Heat Mass Transf 52 (2009) 1794-1801.

[24] J.M. Wu, W.Q. Tao, Numerical study on laminar convection heat transfer in a rectangular channel with longitudinal vortex generator, Part A: verification of field synergy principle, Int J Heat Mass Transf 51 (2008) 1179-1191.

[25] Chemineer, kenics: static mixing technology, Chemineer Inc, 1998 Bulletin 800 (commercial documentation).

[26] H. Mohand Kaci, C. Habchi, T. Lemenand, D. Della Valle, H. Peerhossaini, Flow structure and heat transfer induced by embedded vorticity, Int J Heat Mass Transf 53 (2010) 3575-3584.

[27] T. Lemenand, C. Durandal, D. Della Valle, P. Peerhossaini, Turbulent direct-contact heat transfer between two immiscible fluids, Int J Therm Sci 49 (2010) 1886-1898.

[28] W. Yang, H. Meng, J. Sheng, Dynamics of hairpin vortices generated by a mixing tab in a channel flow, Exp Fluids 30 (2001) 705-722.

[29] D. Dong, H. Meng, Flow past a trapezoidal tab, J Fluid Mech 510 (2004) 219-242.

[30] FLUENT, Inc, FLUENT 6.3 User's guide, (2006).

[31] J.T. Barth, D. Jespersen, The design and application of upwind schemes on unstructured meshes, Proceedings AIAA 27th aerospace sciences meeting, 1989 Reno, Nevada.

[32] R.F. Warming, R.M. Beam, Upwind second-order difference schemes and applications in unsteady aerodynamic flows, Proceedings AIAA 2nd computational fluid dynamics conference, 1975 Hartford, CT.

[33] S.V. Patankar, D.B. Spalding, A calculation procedure for heat, mass and momentum transfer in three-dimensional parabolic flows, Int J Heat Mass Transf 15 (1972) 1787-1806.

[34] H. Mohand Kaci, T. Lemenand, D. Della Valle, H. Peerhossaini, Effects of embedded streamwise vorticity on turbulent mixing, Chem Eng Process 48 (2009) 1457-1474.

[35] M.M. Gibson, B.E. Launder, Ground effects on pressure fluctuations in the atmospheric boundary layer, J Fluid Mech 86 (1978) 491-511.

[36] B.E. Launder, G.J. Reece, W. Rodi, Progress in the development of a Reynolds stress turbulence closure, J Fluid Mech 68 (1975) 537-566.

[37] B.E. Launder, Second-moment closure: present... and future? Int J Heat Fluid Flow 10 (1989) 282-300.

[38] M. Wolfstein, The velocity and temperature distribution of one-dimensional flow with turbulence augmentation and pressure gradient, Int J Heat Mass Transf 12 (1969) 301-318.

[39] H.C. Chen, V.C. Patel, Near-wall turbulence models for complex flows including separation, AIAA J 26 (1988) 641-648.

[40] B. Bonhoffa, S. Parneixa, J. Leuscha, B.V. Johnsona, J. Schabackerb, A. Bölcs, Experimental and numerical study of developed flow and heat transfer in coolant channels with 45 degree ribs, Int J Heat Fluid Flow 20 (1999) 311-319.

[41] R.K. Rahmani, T.G. Keith, A. Ayasoufi, Numerical study of the heat transfer rate in a helical static mixer, J Heat Transf 128 (2006) 769-783.

[42] A. Schaffrath, K.C. Fischer, T. Hahm, S. Wussow, Validation of the CFD code fluent by post-test calculation of a density-driven ROCOM experiment, Nucl Eng Des 237 (2007) 1899-1908.

[43] J.E. Duran, F. Taghipour, M. Mohseni, CFD modeling of mass transfer in annular reactors, Int J Heat Mass Transf 52 (2009) 5390-5401.

[44] T. Defraeye, B. Blocken, J. Carmeliet, CFD analysis of convective heat transfer at the surfaces of a cube immersed in a turbulent boundary layer, Int J Heat Mass Transf 53 (2010) 297-308.

[45] J.H. Lienhard, A heat transfer textbook, third ed., Phlogiston press, Cambridge Massachusetts, 2008, pp. 691-724.

[46] J.O. Hinze, Turbulence, second ed., McGraw-Hill, New York, 1975.

[47] P.J. Roache, Quantification of uncertainty in computational fluid dynamics, Annu Rev fluid Mech 29 (1997) 123-160.

[48] I.B. Celik, U. Ghia, P.J. Roache, C.J. Freitas, H. Coleman, P.E. Raad, Procedure for estimation and reporting of uncertainty due to discretization in CFD applications, J Fluids Eng 130 (2008) 078001.

[49] F.B. Gessner, The origin of secondary flow in turbulent flow along a corner, J Fluid Mech 58 (1973) 1-25.

[50] A. Husert, S. Biringen, Direct numerical simulation of turbulent flow in a square duct, J Fluid Mech 257 (1993) 65-95.

[51] H.S. Choi, T.S. Park, The influence of streamwise vortices on turbulent heat transfer in rectangular ducts with various aspect ratios, Int J Heat Fluid Flow 40 (2013) $1-14$.

[52] C. Habchi, T. Lemenand, D. Della Valle, H. Peerhossaini, Turbulence behavior of artificially generated vorticity, J Turbul 11 (2010) 1-28. 